\title{
Automated Methodology for Optimizing Menus in Personalized Nutrition
}

\author{
Valery I. Karpov ${ }^{1}$, Nikolay M. Portnov ${ }^{2}$ \\ Department "Information Systems and Technologies" \\ K.G. Razumovsky Moscow State University of Technologies \\ and Management (The First Cossack University) \\ Moscow, Russian Federation
}

\author{
Igor A. Nikitin ${ }^{3}$ \\ Department "Technology of Grain Processing, Bakery \\ Pasta and confectionery industries" \\ K.G. Razumovsky Moscow State University of Technologies \\ and Management (The First Cossack University) \\ Moscow, Russian Federation
}

\author{
Yury I. Sidorenko ${ }^{4}$, Igor V. Zavalishin ${ }^{9}$ \\ K.G. Razumovsky Moscow State University of Technologies \\ and Management (The First Cossack University) \\ Moscow, Russian Federation
}

\author{
Sergey M. Petrov ${ }^{5}$ \\ Department "Automated Control Systems" \\ K.G. Razumovsky Moscow State University of Technologies \\ and Management (The First Cossack University) \\ Moscow, Russian Federation
}

\author{
Nadezhda M. Podgornova ${ }^{6}$ \\ Department "Technologies of Production and Organization of \\ Catering and Merchandising" \\ K.G. Razumovsky Moscow State University of Technologies \\ and Management (The First Cossack University) \\ Moscow, Russian Federation
}

\author{
Mikhail Yu. Sidorenko ${ }^{7}$, Sergey V. Shterman ${ }^{8}$ \\ GEON Limited Liability Company \\ Moscow, Russian Federation
}

\begin{abstract}
In the personalized nutrition rationalized management system the central practical task is to compile an optimized menu that provides the best value for a multi-criteria set of assessments. These are nutrient composition, cost (economic acceptability), energy value, food intolerances, individual preferences, etc. To solve the problem, a combined optimization method is used. It includes preliminary ordering of options and controlled enumeration. The result of solving the problem of developing a personalized nutrition menu is a diet that meets the needs of a particular diet, taking into account its nutritional status, individual preferences and intolerances, medical appointments. In connection with discrete values of the outputs of dishes and recipes, the task of optimizing the human diet is in practice a combinatorially integer, and for its solution the method of computer modeling and controlled enumeration of options was used. Evaluation of the effectiveness of optimization is carried out by external experts. The developed menu design system makes it possible to repeatedly solve the problem of optimizing a personalized menu when changing incoming data for reasons of changing dietary tasks, introducing new products, changing food preferences, etc. With this approach, the system is a personalized food model that is regularly used for rational planning and allows to achieve a reduction in labor costs (compared to the "manual" compilation of a menu in a computer system) by 2-3 times. An additional way to use such a model is the targeted design of functional product formulations. Moreover, the properties of the product are not evaluated in isolation, but as part of a specific diet.
\end{abstract}

Keywords-Personalized nutrition; menu optimization; nutrition management; practical nutrition
Managing the nutrition of an individual or an entire team is one of the complex tasks of an interdisciplinary nature, which use knowledge of medical dietetics, technologies of culinary and food production, organic chemistry, psychology and other sciences $[1,2,3,4]$.

In the complex of tasks to be solved in the system of rational nutrition management, the key one is the development of a menu corresponding to medical goals or the goals of meeting the human body's need for energy, nutrients, micronutrients. The implementation of individual characteristics of food perception is also very important. In personalized nutrition [5], such a key task is to compile a set of daily menus for a given period using specific recipes and food outlets for a given composition of meals, menu sections and the total mass of food.

At the moment, optimization calculations are carried out using spreadsheets (which is a form of manual calculation) [3]. In some cases specialized computer systems are used, including means of nutrient calculations [6,7]. Here the menu compilation procedure is implemented in computer forms, calculation of nutrient composition is carried out automatically, the decision on compliance of the developed diet with the initial requirements is made by the technologist who develops the menu, and if necessary, introduces corrections into the next cycle of calculation and evaluation $[8,9]$.

\section{INTRODUCTION}


There are also approaches based on the automatic generation of a diet as a mixture of food products or dishes, which do not provide an appropriate level of consumer quality and, as a result, also require the manual work of a specialist.

Common disadvantages of these solutions are: significant labor costs, duration of work, lack of an objective assessment of options being developed. These drawbacks not allow to realize the possibility of a full-fledged launch of these methods on a massive scale, required for personalized nutrition.

A numerical representation of parameters for evaluating nutrition and its components provides an objective ordering of options, and makes it possible to calculate the measure of proximity of estimates, including the main assessment of the compliance of the actual indicator with the norm. As the main meters in assessing the quality of nutrition, in the sense of its usefulness for the body, the energy value and content of the main (proteins, fats, carbohydrates) and minor (vitamins and minerals) nutrients are used. Many of these indicators will be called nutrients. To assess consumer preferences (for example, taste) and expert ratings, point scales are used. For other indicators, conventional numerical meters are used such as price, weight.

The nutrient composition values of the formulations are determined by the composition of the ingredients [10,11]. Calculation of the nutrient composition of the formulation is carried out according to the formula (1):

$$
H(n)=\sum_{i=1}^{K} N p(i, n) * p(i) *(1-P p(n) / 100)
$$

Where:

$H(n)$ - is the mass of the $n$-th nutrient present in the dish;

$K$ - is the number of ingredients;

$i$ - is the ingredient number;

$P p(i, n)$ - $n$ nutrient content in 100 grams of the $i$ ingredient;

$p(i)$ - is the mass of the $i$-th ingredient in grams;

$P p(n)$ - is the percentage of losses during the processing of the nutrient $n$.

The menu optimization criteria include: compliance of the nutrient composition with an individual consumption standard, standards for natural indicators, cost (economic acceptability), caloric structure by meals, food intolerances, individual preferences, variety, and food compatibility.

Additional estimates (indicators): balance of proteins/fats/carbohydrates by weight and share in calories; protein quality (amino acid rate [12] as well as utilization coefficient, balance coefficient, index of essential amino acids $[3,13,14,15,16])$; quality of the fatty acid composition (ratio of fatty acids $[17,18]$ ); achievement of target designations for minor nutrients [1]; variability of values by days (permissible unevenness). In some cases, individual indicators can become part of optimization criteria, for example, for a ketogenic diet [19], an increased proportion of fat and a decrease in the proportion of carbohydrates are required.
Accepted axioms: food, diet - a set of complexes of dishes (not a set of products); specificity of norms (indication of average target values); determination of menu parameters by a set of days (not per day or a separate meal); the concept of "consistent with the norm" (a measure of proximity to the norm, an optional match); limiting the nutritional model just by consumption (excluding the digestibility by the body, the actual properties of raw materials and cooking); ignoring the combined effects of food components; restriction of the list of methods for evaluating nutrition only having a scientific justification.

The aim of the study was to develop a computer system prototype that simulates the development of a personalized menu, including its optimization according to a multi-criteria set of indicators, to test the methods of the most automated development of personalized diets. The computer system includes real data sets on food recipes, the nutritional composition of products and recipes, practical nutritional calculation algorithms, diet quality assessments according to certain criteria (protein biological value, fat quality, etc.) for the most adequate reproduction of the traditional menu development process. Modeling the development process in various conditions (taking into account the individual characteristics of eating, differences in standards for categories of eating, differences in assessment methods) is necessary to develop a methodology for developing diets, to achieve the maximum degree of automation, to check the effectiveness of optimization tools.

\section{STATEMENT AND ANALYSIS OF THE PROBLEM OF MENU VARIANTS DESIGN WITH GIVEN CHARACTERISTICS}

The result of solving the problem of developing a personalized nutrition menu is a diet that meets the needs of a particular diet, taking into account its nutritional status, individual preferences and intolerances, medical appointments. The degree of closeness of indicators of the actual diet to a predetermined need is estimated in numerical terms (objective function), taking into account a number of criteria (particular assessment parameters): nutrient standards, balance of basic nutrients, cost, consumer assessment, protein quality, quality of fats, uniformity of indicators for days and meals; the mutual relation of particular criteria is determined by weight coefficients for the components of the objective function, which can vary in accordance with a specific situation.

The developed diet is described by a set of technological documentation, including a menu for a period (week or more) with an indication of specific recipes for meals combined for day meals including dish outcome. The recipes used in the menu are documented by routings. The documentation set also includes the used product range, information on the nutritional composition of foods and dishes, nutritional calculations, reflecting the nutritional structure by days and meals, and evaluating the variability of numerical indicators, calculations of compliance of the diet with consumption standards.

The initial data is: 1) objective data on the person anthropometric measurements, medical history and medical appointments (in terms of nutrition), the study of actual nutrition, body composition data; 2) information on individual preferences and intolerances of products and dishes; 3) official 
consumption standards, scientifically based methods for assessing food quality (amino acid rate, fatty acid composition, balance of proteins/ fats/ carbohydrates, etc.); 4) information from reference scientific literature on formulations, nutrient composition.

Directly for the optimization problem, the source data is a set of values of individual nutritional appointments (a vector of values for a set of nutrients) that reflects characteristics of personalized nutrition (in contrast to the classical task of developing collective/average nutrition).

Due to the discrete values of the outputs of dishes and recipes, the nature of human nutrition in the form of complexes of dishes, taking into account their mutual influence, the task of optimizing the human diet in practice is a combinatorially integer. To solve it controlled enumeration of options and computer simulation methods are applied rather than analytical mathematical methods.

\section{COMPREHENSIVE OPTIMIZATION ALGORITHM FOR THE DEVELOPMENT OF THE MENU WITH THE GIVEN CHARACTERISTICS OF NUTRITION VALUE}

To determine the optimal value on the set of menu options, an integral estimate (convolution) - Q is used, a linear function with weight coefficients that reflect the significance of each individual component of the estimate (2):

$Q=\sum_{i=1}^{N} B k r(i) * Z(i)$

Where:

$N$ - is the number of criteria;

$B k r(i)$ - the value of the assessment in points according to the $i$-th criterion normalized on a common scale (0-10);

$\mathrm{Z}(\mathrm{i})$ is the weight of the $i$-th criterion that determines its importance, $0<=Z$ (i) $<=1$.

The use of a simplified linear objective function is explained by the insufficient amount of scientific knowledge to build more flexible models and is a primary approximation. To justify the weight coefficients of the integral function, an expert approach is used; a more reasonable estimate can be obtained by the method of hierarchy analysis [20].

Because of a large set of heterogeneous initial requirements for the menu optimization algorithm, the requirement of model stability (algorithm convergence) is presented to obtain the optimal solution even with a possible initial formal incompatibility of conditions when the found solution provides the closest approximation to a given set of conditions.

To ensure stability, a combined optimization method is used. Preliminary optimization includes: clustering the initial set of dishes to reduce the dimension of the task; selection of candidate dishes; ordering lists of dishes in the direction of local optimization by individual nutrients that are main components of optimization. Final optimization is performed by combinatorial sorting according to pre-ordered lists of dishes, in combination with local optimization for maximum compliance with the norms (partial change of the bookmark norms within the permissible limits, outputs across the cluster of permissible values). This method, in addition to stability, also provides practical results with limited time. With an increase in the search time, in the general case, the value of the integral estimation function is improved (practical convergence).

Prior to optimization, a check is carried out for completeness of the initial data and compatibility of conditions with the possibility of reformulating mutually exclusive requirements. An example of such "optimization of the initial data" is the rejection of the use of natural norms that are incompatible with the norms for nutrients and their transfer from the category of optimization criteria to indicators. The reasons for such inconsistencies may be inconsistency in official standards, errors in determining individual consumption standards.

For practical implementation in the form of a computer program, the rapid development tool "Platform 1C: Enterprise" (version 8.3) is used. For editing of recipes the "Technological chart" module is used; for editing the menu and related calculations the "Development of ration" module from the program "1C: Medicine. Dietary nutrition" [7] is used. As a supplier of raw data for dishes - electronic recipe collections in the format of "Recipe-3" is used. The module "Development of the diet" is also used to describe the source data for subsequent optimization: the menu template (meals and sections), the composition of the food components, the distribution of calories in meals. Nutrient consumption standards are filled in the directory "Categories of persons", the substantiation for individual consumption standards is carried out in the computer system "Monitoring of physical development and nutritional status" [21].

To solve optimization problems, a standard 1C system (supplied with source codes) was amended:

- Features of the diet for personalized nutrition are implemented in the form of directories of personal preferences and intolerances.

- For reference sets of individual recipes the "Dishes" reference book was modified.

- To visualize a set of estimates of a particular menu option, the report "Assessment of a menu option" is used, which also contains means for decrypting (explicating) the calculated values.

- Optimization of the menu ("processing" in terms of the $1 \mathrm{C}$ platform) is carried out by the "Menu Optimization" subprogram, which includes the stages of preliminary optimization (ordering of the original lists of dishes), enumeration of menu variants, calculation of private estimates and the value of the integral function (final optimization). To control the convergence and the possibility of the resumption of enumeration, a convoluted identification of options and a variant assessment log are used. 
- To visualize the progress of evaluating a number of options, the "Report on menu options" report has been developed.

- To control the composition of optimization parameters and composition of indicators, the parameter settings form is used, in which weight coefficients of the integral objective optimization function are also introduced (see Fig. 1 and 2).

To assess the effectiveness of the optimization machine, an external expert assessment of the quality of complexes of dishes in one meal, the compatibility and variety, the correspondence of the actual menu indicators and norms (proximity measures) is used. In addition to the composition of the complexes of dishes, i.e. the menu itself experts also use calculated indicators that are not involved in optimization such as uniformity of values by day, balance. For convenient and informative perception of such information, in addition to numerical arrays, means of infographics and decoding of the final values are used. The accumulated expert assessments are used to train optimization tools (they are used at the preliminary optimization stage) in subsequent work sessions. Experts in the fields are involved in evaluating effectiveness: medicine (assessment of the impact on health and treatment), food preparation technology (practical feasibility, risks), as well as the consumer himself.

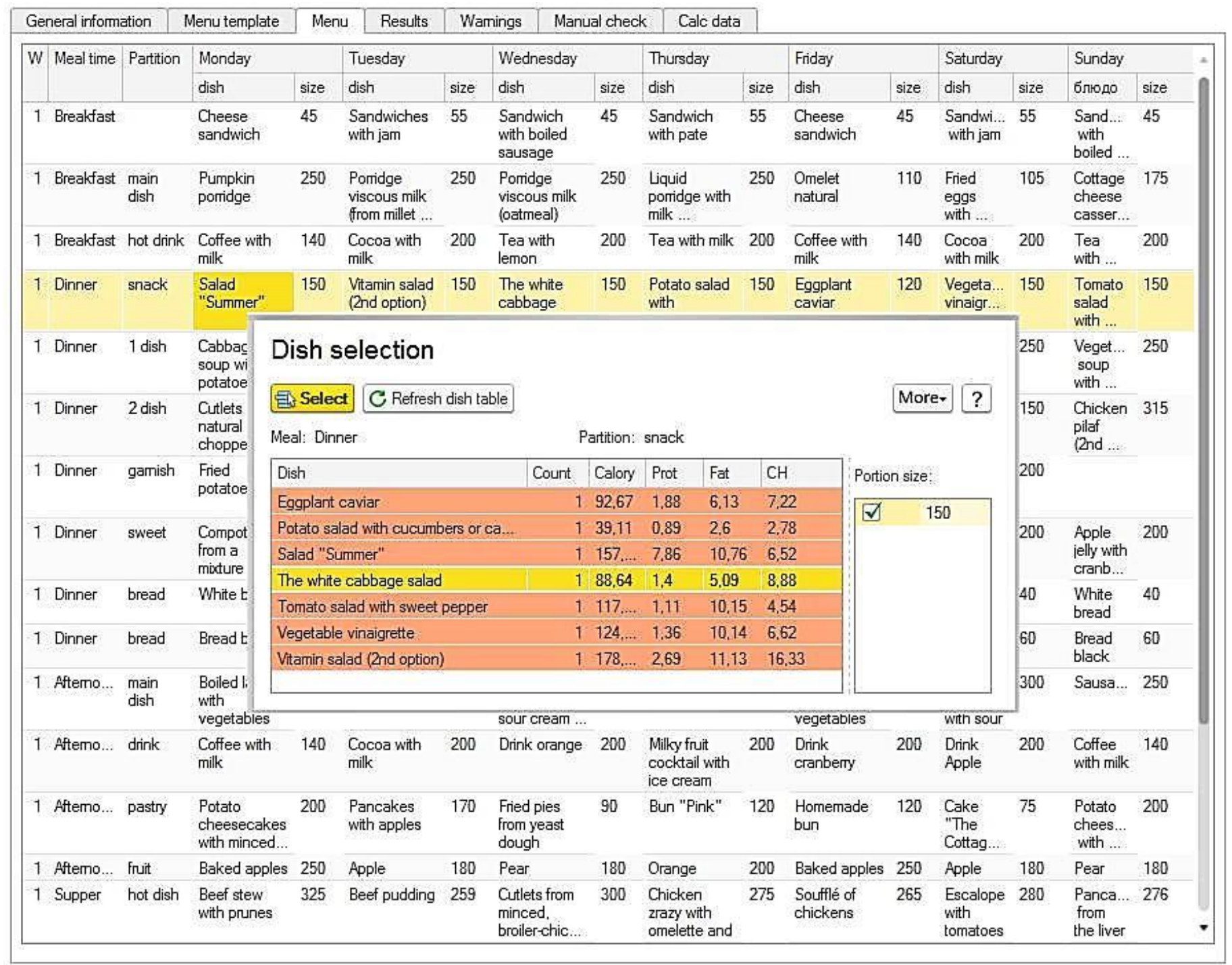

Fig. 1. Interface of the Menu Editor in the Module "Development of the Ration". 


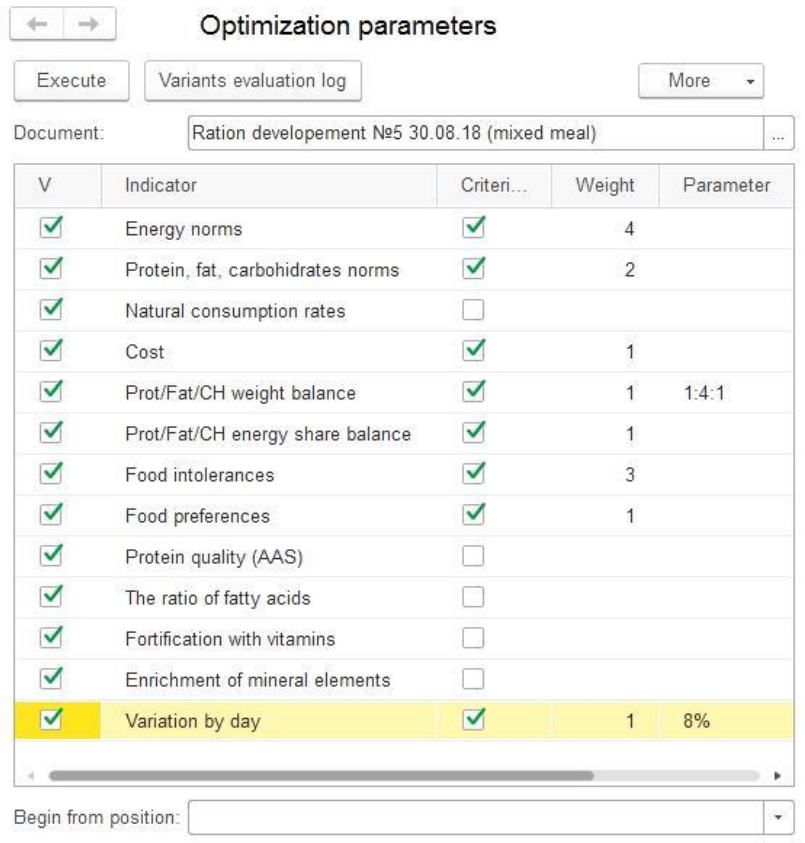

Fig. 2. Parameters of the Optimization Module.

\section{IMPLEMENTATION OF THE PROGRAM DISCUSSION OF RESULTS}

The developed system has been tested for cases of compiling a personalized menu (individual and micro-teams) for a healthy person and patients with alimentary-dependent diseases (with the indicated diets, medical prescriptions), taking into account individual preferences in dishes, for individually calculated nutritional needs (ICS - individual consumption standards were determined using the NutriMon computer system [21]) with various correlations of importance of particular criteria of the integral objective function. Based on the results of testing, changes were made to the system. The new version takes into account individual characteristics of recipes and cooking methods, typical servings, sets of products with individual binding. By means of automatic optimization in comparison with the "manual" menu making in a computer system a reduction in labor costs was achieved by 2,3 times.

The system provides the ability to repeatedly solve the problem of optimizing a personalized menu when changing incoming data for reasons of changing dietary tasks, introducing new products, changing food preferences, etc. With this approach, the menu design system is a personalized food model that is regularly used for rational planning.

An additional way to use such a model is the targeted design of functional product formulations, including the case of targeted development for personalized nutrition. Moreover, the properties of the product are not evaluated in isolation, but as part of a specific diet.

Directions for the development of the model: improvement of primary information about dishes and recipes and their official publication; taking into account the variability (statistical nature) of the evaluation indicators; comparison of actual consumption with a planned menu as part of an overall assessment of the effectiveness of diet therapy; use of nutribiomics and nutrigenomics; psychology of food consumption; family model of nutrition (optimization on a microgroup, taking into account individual characteristics); analysis of the structure of a multi-criteria set of assessment indicators to identify the main components; porting to a mobile platform and to a cloud service.

\section{CONCLUSION}

Software has been developed for the automated development of personalized diets with the possibility of automatic optimization to achieve maximum proximity of the menu indicators to the desired nutritional settings. Evaluation of the quality of the developed diet is carried out according to a set of criteria using a generalizing (integral) function, the behavior of which is adjusted by a set of component importance coefficients.

The result was achieved using: ready-made computer programs " $1 \mathrm{C}$ : Planned Nutrition" and "NutriMon" (monitoring of nutritional status and physical development); electronic collections of recipes in the "RecipeBook" format; development of additional optimization modules and database modification by means of the 1C: Enterprise platform.

The materials for state registration of the developed computer program are prepared.

Further directions for the development of the model are: improving the primary information about dishes and recipes and their official publication; taking into account the variability (statistical nature) of the evaluation indicators; comparison of actual consumption with a planned menu (as part of an overall assessment of the effectiveness of diet therapy); use of nutribiomics and nutrigenomics; psychology of food consumption; family model of nutrition (optimization on a microgroup, taking into account individual characteristics); analysis of the structure of a multi-criteria set of assessment indicators to identify the main components; porting to a mobile platform and to a cloud service.

\section{ACKNOWLEDGMENT}

This article was written with support from the Government of the Russian Federation (Resolution of 16.03.2013), agreement and subsidies for the fulfillment of a fundamental part of a state order under Project 15.9195.2017/5.1.

\section{REFERENCES}

[1] Methodological recommendations 2.3.1.2432-08 Norms of physiological requirements for energy and nutrients for various population groups of the Russian Federation.

[2] Baranovsky A.Yu., Dietology, St. Petersburg: Peter, 2017.

[3] Lisin P.A., Computer modeling of production processes in the food industry, St. Petersburg: Lan, 2016.

[4] Nikitina M.A., Chernukha I.M., "Multicriteria optimization of the formulation of the product," Theory and practice of meat processing; 3 (3): 89-98.DOI 10.21323 / 2414-438X 2018-3-3-89-98, 2018.

[5] Sidorenko M.Yu., Personified Nutrition, M .: DeLi Plus, 2016.

[6] Krutko V.N. et al., "Evaluation and optimization of nutrition using the computer system "Nutrition for Health and Longevity"/ "Computer Science of Health and Longevity". Proceedings of the ISA RAS. Vol. 13. Moscow.: KomKniga., 2005.

[7] Computer program "1C: Medicine. Dietetic nutrition", On the Internet: http: // http://diet.1cp.ru/ Date of access 04/15/2019. 
[8] Mosov A.V., Portnov N.M. The methodology for the development of collective diets, Moscow: Eidos, 2015.

[9] Barry C et al, Optimization of the Order Menu in the Electronic Health Record Facilitates Test Patterns Consistent With Recommendations in the Choosing Wisely Initiative / Am J Clin Pathol. 2019 Aug 1. doi: 10.1093/ajcp/aqz134.

[10] Reinivuo H., Laitinen K., Proposal for the harmonization of recipe calculation procedures, EoroFIR, 2007.

[11] Vasquez-Caicedo et al., Report on collection of rules on use of recipe calculation procedures including the use of yield and retention factors for imputing nutrient values for composite foods, EuroFIR, 2008.

[12] FAO food and nutrition paper 92. Dietary protein quality evaluation in human nutrition/ Report of an FAO Expert Consultation, Geneva: FAO, 2013.

[13] Lisitsyn A.B., Nikitina M.A., Sus E.B., "Evaluation of the quality of protein using computer technology" Food industry. - 2016. - No. 1. P.26-29.

[14] Muratova E.I. et al., Automated design of complex multi-component food products, Tambov: FSBEI HPE “TSTU”, 2011.
[15] Musina O.N., Computer design of recipes, M.: Direct Media, 2015.

[16] Nadtochy L.A., Orlova O.Yu., Innovations in biotechnology. Part 2. Food Combinatorics: Educational Method. manual, St. Petersburg: ITMO University, 2015.

[17] FAO Food and nutrition paper 91, Fats and fatty acids in human nutrition. Report of an expert consultation, Geneva: FAO, 2011.

[18] Simonopolus A.P., Visioli F., More on Mediterranean Diets, Basel: KARGER, 2007.

[19] Kossoff E.H. et al., «Optimal clinical management of children receiving dietary therapies fir epilepsy: Updated recommendations of the International Ketogenic Diet Study Group» Epilepsia Open, 3(2): 175192, 2018.

[20] Nikitina M. et al., «Application of the Hierarchy Analisys Method at the Foodstuff Quality Evaluation» International Journal of Advanced Computer Science and Applications, т. 9, № 5, pp. 51-59, 2018.

[21] Computer program "Monitoring of physical development and nutritional status", on the Internet: http://www.1cp.ru/solutions/NutriMon/ Date of access April 15, 2019. 